\title{
The Transcription Factor Bach1 Suppresses the Developmental Angiogenesis of Zebrafish
}

\author{
Li Jiang, ${ }^{1}$ Meng Yin, ${ }^{2}$ Jie Xu, ${ }^{1}$ Mengping Jia, ${ }^{1}$ Shaoyang Sun, ${ }^{3}$ Xu Wang, \\ Jianyi Zhang, ${ }^{4}$ and Dan Meng ${ }^{1}$ \\ ${ }^{1}$ Department of Physiology and Pathophysiology, School of Basic Medical Sciences, Fudan University, Shanghai 200032, China \\ ${ }^{2}$ Department of Cardiothoracic Surgery, Shanghai Children's Medical Center, Shanghai Jiao Tong University School of Medicine, \\ Shanghai 200127, China \\ ${ }^{3}$ Department of Biochemistry and Molecular Biology, School of Basic Medical Sciences, Fudan University, Shanghai 200032, China \\ ${ }^{4}$ Department of Biomedical Engineering, School of Medicine, University of Alabama at Birmingham, Birmingham, AL 35294, USA
}

Correspondence should be addressed to Dan Meng; dmeng@fudan.edu.cn

Received 20 January 2017; Accepted 23 February 2017; Published 14 March 2017

Academic Editor: Veronika A. Myasoedova

Copyright (C) $2017 \mathrm{Li}$ Jiang et al. This is an open access article distributed under the Creative Commons Attribution License, which permits unrestricted use, distribution, and reproduction in any medium, provided the original work is properly cited.

Bachl disrupts Wnt/ $\beta$-catenin signaling, reduces the proliferation, migration, and tube formation activity of endothelial cells (ECs), and suppresses angiogenesis in mice with surgically induced hind-limb ischemia (HLI). However, the function of Bachl during developmental angiogenesis in zebrafish remains unclear. Here, we found that zebrafish Bachl was expressed ubiquitously during early embryonic development in zebrafish. Bachlb mRNA injection of $T g(f l i 1: g f p)$ fish disrupted intersegmental vessels (ISV) and dorsal longitudinal anastomotic vessels (DLAV) and suppressed endogenous Wnt/ $\beta$-catenin signaling and Wnt8a stimulated vascular endothelial growth factor (VEGF) and interleukin-8 (IL-8) gene expression at early embryonic stages of zebrafish. Furthermore, chromatin immunoprecipitation experiments confirmed that Bachl occupied the TCF/LEF-binding site of the VEGF promoter in human umbilical vein endothelial cells (HUVECs). Bachl inhibited VEGF transcription by recruiting histone deacetylase 1 (HDAC1) to the VEGF promoter in HUVECs. Exogenous administration of VEGF or IL-8 partially rescued Bachl-driven antiangiogenic functions in HUVECs. Taken together, these observations indicate that Bachl suppresses the developmental angiogenesis of zebrafish and that this function is associated with declines in Wnt/ $\beta$-catenin signaling and VEGF and IL- 8 expression.

\section{Introduction}

Wnt signaling has been implicated in cardiovascular development, including placental vascular development, endothelial cell fate specification and endothelial cell proliferation, and vascular growth and integrity [1-3]. Activation of Wnt signaling was observed during embryonic development in many types of vessels [1], and endothelial-specific deletion of $\beta$ catenin affected the development of the embryonic vasculature and resulted in early lethality in utero of mouse [4]. Disruption of Wnt signaling in zebrafish resulted in severe vascular defects during embryonic angiogenesis [3, 5]. Wnt signaling is also reported to promote expressions of vascular endothelial growth factor (VEGF) and interleukin-8 (IL-8) $[6,7]$, which have been shown to have angiogenic activity in human endothelial cells and zebrafish [8-11].
BTB and CNC homology 1 (Bach1) is a basic leucine zipper transcription factor that heterodimerizes with small Maf proteins and binds to the antioxidant responsive element (ARE) to repress the expression of genes encoding antioxidant proteins $[12,13]$. We have shown that Bachl binds to TCF4, reduces the interaction of $\beta$-catenin with TCF4, and disrupts Wnt/ $\beta$-catenin signaling by recruiting histone deacetylase 1 to the promoter of TCF4-targeted genes [14]. Our results also indicate that Bachl reduces the proliferation, migration, and tube formation activity of human endothelial cells (ECs), induces endothelial cell apoptosis and cell-cycle arrest, and suppresses angiogenesis in mice with surgically induced hind-limb ischemia (HLI) [14, 15]. These observations suggest Bachl as a major regulator of endothelial function and ischemia-induced angiogenesis. However, little is yet known about the role of Bachl in vascular development. 
Bach1 is conserved in vertebrates and some urochordates [16]. In zebrafish, two Bach1 homolog genes (Bachla; Bachlb) have been reported and Bachlb is the main transcription factor, which heterodimerized with MafK to repress target gene expression, including heme oxygenase-la (hmoxla) and zymogen [16-18]. Thus, in the present study, zebrafish was used as an in vivo model to study the role of Bachlb in Wnt/ $\beta$ catenin signaling and the developmental angiogenesis, and in vitro cell transfection and chromatin immunoprecipitation (ChIP) assays were conducted to elucidate how Bachl regulates Wnt target gene (VEGF) expression in human umbilical vein endothelial cells (HUVECs).

\section{Methods and Materials}

2.1. Maintenance of Zebrafish. The zebrafish AB line was used, and embryos and adult fish were raised according to the standard protocol described in The Zebrafish Book. $\mathrm{Tg}\left(\right.$ flil:EGFP $^{y 1}, \mathrm{Tg}(\mathrm{TOP}: \mathrm{GFP})^{w 25}$, and $\mathrm{Tg}$ (hsp701:wnt8aGFP $)^{w 34}$ transgenic zebrafish lines [19-21] were provided by Dr. Xu Wang (Fudan University). Embryos were obtained by natural spawning and were maintained in E3 zebrafish water at $28.5^{\circ} \mathrm{C}$ and staged according to Kimmel et al. [22]. Embryos were heat-shocked at the indicated stage by transferring them into egg water prewarmed to $38^{\circ} \mathrm{C}[\mathrm{Tg}$ (hsp701:wnt8aGFP $)^{w 34}$ ] and kept at this temperature for 25 minutes. After heat-shock, the plate containing the embryos was transferred back into a $28.5^{\circ} \mathrm{C}$ incubator. Experimental procedures and animal use and care protocols were approved by the Fudan University Animal Ethics Committee and were consistent with the "Guide for the Care and Use of Laboratory Animals" published by the National Institutes of Health (NIH) of the United States.

2.2. mRNA Synthesis. Zebrafish Bachlb gene was amplified through reverse transcription-polymerase chain reaction (RT-PCR) and verified by DNA sequencing. DNA generated by PCR can be transcribed directly from the PCR provided it contains a T7 RNA polymerase promoter upstream of the sequence to be transcribed. Then zebrafish Bach $1 b$ mRNAs were synthesized from linearized templates using the mMessage mMachine kit (Invitrogen, Carlsbad, CA). Zebrafish embryos were injected at the one cell stage with $200 \mathrm{pg}$ of synthetic Bach $1 b$ mRNA in water.

2.3. Whole Mount Antibody Staining. Zebrafish embryos were fixed two hours in 4\% paraformaldehyde (PFA)/phosphatebuffered saline (PBS), washed, dehydrated in methanol, and stored at $-20^{\circ} \mathrm{C}$. Embryos were rehydrated, permeabilized with proteinase $\mathrm{K}$, and fixed again with $4 \% \mathrm{PFA} / \mathrm{PBS}$. After blocking in $1 \%$ bovine serum albumin (BSA) plus $2 \%$ serum in PBS, embryos were incubated with an anti-Bachl antibody (ab115210, Abcam, Cambridge, MA) at $4^{\circ} \mathrm{C}$ overnight. On the following day embryos were washed and the secondary antibody was added at $4^{\circ} \mathrm{C}$ overnight. The color reaction was developed with the avidin-biotin-horseradish peroxidase method (ABC kit) and diaminobenzidine (DAB) as a chromogen.
TABle 1: Primers used for qPCR and ChIP.

\begin{tabular}{lc}
\hline Primer & Sequence $\left(5^{\prime}-3^{\prime}\right)$ \\
\hline Bachlb-F (zebrafish) & CTTGCTGGAGTTCGCCTACA \\
Bachlb-R (zebrafish) & AATGTACCTCTGTTGCTGTCA \\
IL-8-F (zebrafish) & AAGCCGACGCATTGGAAAAC \\
IL-8-R (zebrafish) & AGGGGTCCAGACAGATCTCC \\
VEGF $_{165}-\mathrm{F}$ (zebrafish) & ATCGAGCACACGTACATCCC \\
VEGF $_{165}-\mathrm{R}$ (zebrafish) & CCTTTGGCCTGCATTCACAC \\
GAPDH-F (zebrafish) $_{\text {GAPDH-R (zebrafish) }}$ & GAGGCTTCTCACAAACGAGGA \\
Human VEGF ChIP & TGGCCACGATCTCCACTTTC \\
(-301/-105)-F & CACTTTCCTGCTCCCTCCTC \\
Human VEGF ChIP & \\
$(-301 /-105)-R$ & AGCCTCAGCCCTTCCACA \\
Human VEGF ChIP & \\
(-1589/-1357)-F & GAGGCTATGCCAGCTGTAGG \\
Human VEGF ChIP & \\
(-1589/-1357)-R & CCCTTTTCCTCCAACTCTCC \\
Human VEGF & \\
Human VEGF & ATCTTCAAGCCATCCTGTGTGC \\
Human GAPDH-F & CAAGGCCCACAGGGATTTTC \\
Human GAPDH-R & CCATCTTCCAGGAGCGAGATC \\
\hline
\end{tabular}

2.4. Quantitative Real-Time Reverse Transcription-Polymerase Chain Reaction. The expression levels of various genes in zebrafish embryos or HUVECs were analyzed by real-time RT-PCR as described previously [23]. Eight to ten zebrafish embryos were collected. Total RNA was extracted using the TRIzol reagent (Invitrogen, Carlsbad, CA) according to the manufacturer's instructions. cDNA was synthesized using SuperScript Reverse Transcriptase (Fermentas, Glen Burnie, MD). RT-PCR analysis was carried out using SYBR Green PCR master mix (Toyobo, Japan). Primers are shown in Table 1. All samples were analyzed using a Bio-Rad real-time analyzer (Bio-Rad Laboratories, Hercules, CA) and results were normalized to the glyceraldehyde-3-phosphate dehydrogenase (GAPDH) expression. Experiments were done at least in triplicate.

2.5. Confocal Microscopy and Imaging. Zebrafish embryos were examined by confocal microscopy; the embryos were embedded live in 1\% low-melting-point agarose (SigmaAldrich, St. Louis, MO) and photographed by a Leica SP8 confocal microscopy system (Bio-Rad, Hercules, CA). Other fluorescent images were taken under Olympus MVX10 (Olympus Corporation) and the fluorescence intensity was quantified using ImageJ software (National Institutes of Health, Bethesda, MA).

2.6. Cell Culture and Tube Formation Assay. Primary human umbilical vein endothelial cells (HUVECs) were obtained from fresh umbilical cord veins from normal pregnancies, and informed consent was received from the pregnant women; a procedure that was approved by the ethics review 
board of Fudan University. HUVECs were isolated by collagenase digestion and identified as previously described [14]. The recombinant adenoviruses encoding human Bachl gene (AdGFP-Bach1) or GFP Control (AdGFP) were purchased from Genechem (Shanghai, China) and used to infect the ECs. For the transduction, a multiplicity of infection (MOI) of 25 was used. No detectable cellular toxicity was observed. HUVECs were cultured in presence of VEGF-A $(50 \mathrm{ng} / \mathrm{mL}$, PeproTech Inc, Rocky Hill, NJ) or IL-8 (50 ng/mL, PeproTech Inc, Rocky Hill, NJ) in a 24 -well plate coated with $200 \mu \mathrm{L}$ of growth factor-reduced Matrigel. Tube length was quantified after 12 hours by measuring the cumulative tube length in five random microscopic fields with an inverted phase contrast microscope using Image software (National Institutes of Health, Bethesda, MA). The relative tube lengths were expressed as the fold change relative to the Control. Each experiment was repeated four independent times.

2.7. Transient Transfection and Luciferase Assay. Transient transfection and luciferase assay were performed as described previously [24]. A $2.65 \mathrm{~kb}$ human VEGF gene promoter was kindly provided by Dr. Jiang (Thomas Jefferson University, USA). VEGF promoter fragments of different sizes (-529 and -228) were amplified from human genomic DNA with appropriate sets of primers. PCR products were cloned into the luciferase vector pGL3-basic. All constructs were verified by sequencing. Cells were transfected with DNA or siRNA together with the indicated VEGF reporter or pGL3basic luciferase reporter and $\beta$-galactosidase plasmid using Lipofectamine 2000. The transfected cells were cultured for 24 hours. Trichostatin A (TSA, T1952, Sigma-Aldrich, St. Louis, MO) was added for 24 hours. Cells were harvested and measured with a luciferase assay kit (Promega, Madison, WI). Relative luciferase activity was calculated as the ratio of Luc/ $\beta$-galactosidase activity and expressed as the ratio of VEGF reporter/pGL3-basic. Each experiment was repeated three independent times.

2.8. Chromatin Immunoprecipitation (ChIP). The assay was performed according to the manufacturer's protocol (ChIPIT protein G magnetic beads kit, Active Motif, Carlsbad, CA). In brief, cells were seeded onto a $10 \mathrm{~cm}$ culture dish and one day later were cross-linked by $1 \%$ formaldehyde for 10 minutes at room temperature and followed by fragmentation of genomic DNA using a sonification apparatus. Cell lysates were used for chromatin immunoprecipitation using Bachl (sc-14700, Santa Cruz, CA), HDAC1 (sc-7872, Santa Cruz, CA), Ac-Histone H3 (sc-56616, Santa Cruz, CA), and Control antibody (sc-2028, Santa Cruz, CA). The protein G magnetic beads-antibody/chromatin complexes were washed and the antibody/chromatin complexes were subsequently eluted. The cross-linked protein/DNA complexes were detached at $65^{\circ} \mathrm{C}$ for four hours followed by purification of the genomic DNA. PCR primers amplifying human VEGF promoters are shown in Table 1. Real-time PCR was carried out using SYBR Green PCR master mix. The amount of DNA associated with each protein (relative to the total amount of DNA used) was determined.
2.9. Western Blotting. Western blotting was performed as described previously [25]. Briefly, cells or tissue homogenates were lysed with SDS sample buffer on ice for 10 minutes. Samples were heated at $95^{\circ} \mathrm{C}$ for five minutes. SDS-PAGE was performed and proteins were detected using antibodies to Bach1 (sc-14700, Santa Cruz, CA) and $\beta$-actin (A5316, SigmaAldrich, St. Louis, MO). Chemiluminescence was detected on a Tanon-5500 Imaging System (Tanon Science \& Technology Ltd, Shanghai, China).

2.10. Measurement of Secreted VEGF via ELISA. Human VEGF-A was quantified in the cell culture supernatant using ELISA assay kit from R\&D Systems, Inc. (Minneapolis, MN) as described previously [23]. Briefly, ECs were transfected with siRNA or transduced with adenovirus. After 24 hours, cells were washed with phosphate-buffered saline (PBS) and fresh medium was added. Supernatants of the cells were collected at 48 hours and the number of cells was counted. An ELISA assay was performed according to the manufacturer's protocol. After measuring the VEGF-A content in the supernatants, the amount of VEGF-A per $10^{6}$ cells was calculated. Each experiment was repeated three independent times.

2.11. Statistical Analysis. Data are expressed as mean \pm SEM from in the Figures. Differences among groups were determined using Prism software with one-way analysis of variance followed by Bonferroni post hoc test. Differences between two groups were assayed by two-tailed Student's $t$ test. The 0.05 level of probability was used as the criterion of significance.

\section{Results}

3.1. Bach1 Was Ubiquitously Expressed during Early Embryonic Development in Zebrafish. We observed that zebrafish Bach1 expression was evident as early as $4 \mathrm{hpf}$ (hours after fertilization) and maintained up to $24 \mathrm{hpf}$ and ubiquitously expressed during early embryonic zebrafish development (Figure 1).

3.2. Overexpression of Bach1b Impairs the Developmental Angiogenesis of Zebrafish. Bachlb plays an important role in Nrf2-MafK pathway in zebrafish [17]. Thus, we overexpressed Bachlb in zebrafish embryos to investigate the role of Bachl during vascular development. Injection of Bachlb mRNA into the one cell stage of $\mathrm{Tg}(f l i 1: E G F P)$ fish embryos resulted in $\approx 2$-fold increase in mRNA expression of Bachlb at $32 \mathrm{hpf}$ as evidenced by qRT-PCR (Figure 2(b)). No detectable apoptosis and toxicity were observed after injection of Bachlb mRNA with $200 \mathrm{pg}$ for each embryo (data not shown). The analysis of the vasculature revealed that intersegmental vessels (ISV) and the dorsal longitudinal anastomotic vessels (DLAV) had not formed properly in Bachlb-overexpressing zebrafish (Figures 2(a) and 2(c)). These results suggest that Bachlb overexpression impairs the developmental angiogenesis of zebrafish embryos.

3.3. Bach1 Suppresses Endogenous Wnt/ $\beta$-Catenin Signaling and Exogenous Wnt8a Stimulated VEGF and IL-8 Gene Expression in Zebrafish. Recently, we have shown that Bach1 


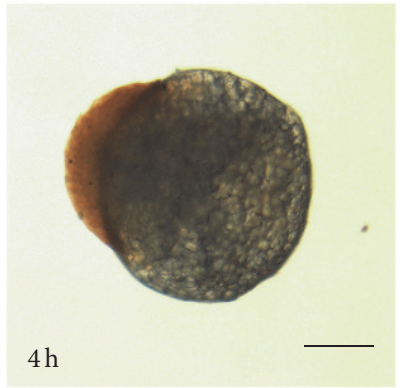

(a)

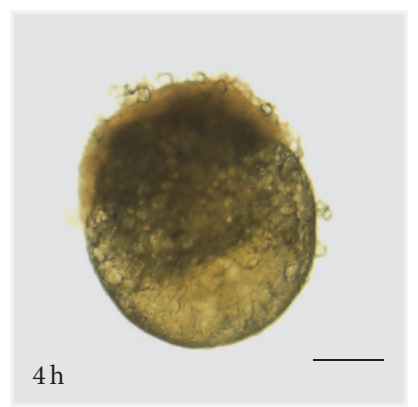

(d)

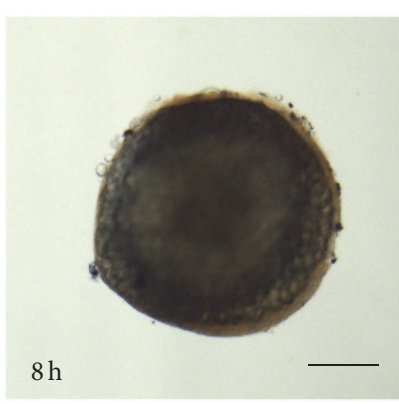

(b)

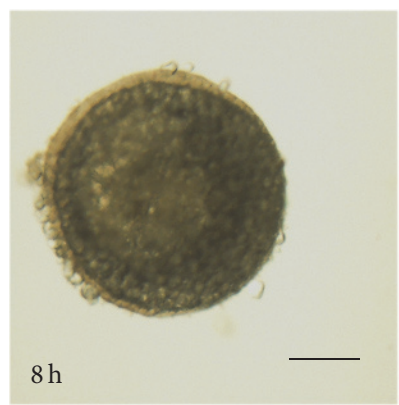

(e)

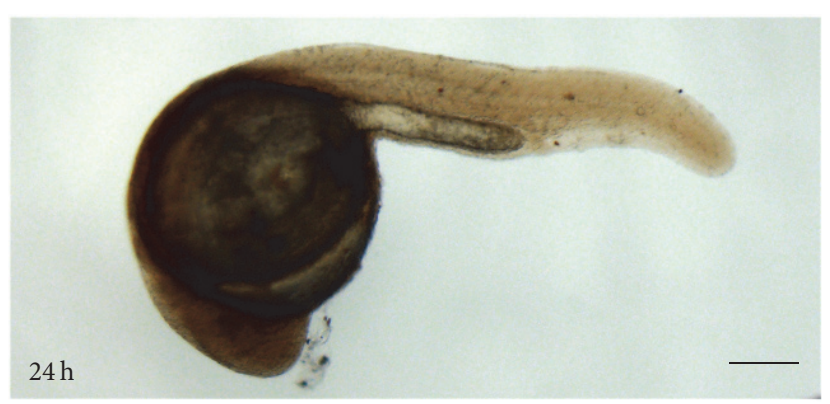

(c)

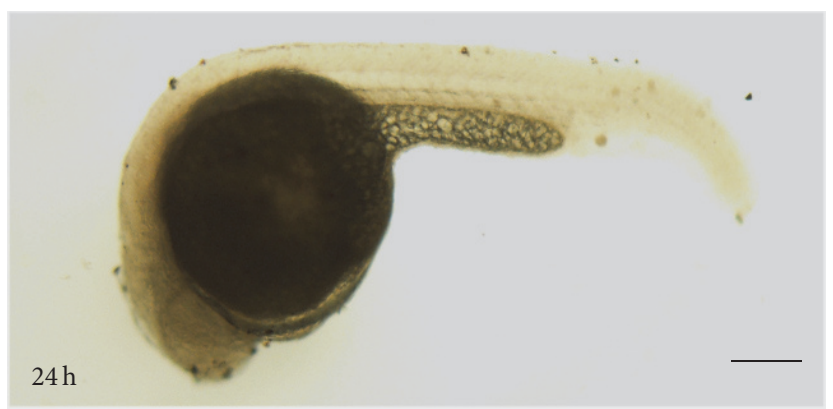

(f)

FIGURE 1: The expression of Bach1 during embryonic development in zebrafish. Bachl expression was evaluated in zebrafish embryos that had been stained with $(\mathrm{a}-\mathrm{c})$ or without $(\mathrm{d}-\mathrm{f})$ a Bachl antibody at $4 \mathrm{~h}(\mathrm{a}, \mathrm{d}), 8 \mathrm{~h}(\mathrm{~b}, \mathrm{e})$, and $24 \mathrm{~h}(\mathrm{c}, \mathrm{f})$ after fertilization. Scale bar: $100 \mu \mathrm{m}$.

disrupts Wnt/ $\beta$-catenin signaling in HUVECs [14]. We then investigated whether Bachlb inhibits $\mathrm{Wnt} / \beta$-catenin signaling at early embryonic stages of zebrafish. We made use of the $\operatorname{Tg}(T O P: G F P)$ transgenic line of zebrafish in which $\beta$ catenin dependent transcriptional activity can be monitored by expression of a GFP reporter driven from a promoter containing multiple TCF/LEF elements [20]. Analysis of TOP:GFP expression showed that a significant decrease in fluorescence intensity with GFP at 36 hours after injection of Bachlb mRNA (Figures 3(a) and 3(b)), indicating that Bachlb suppresses endogenous Wnt/ $\beta$-catenin activity in zebrafish. We then used heat-shock line (hsp:wnt8a) to activate Wnt/ $\beta$ catenin signaling pathway. Heat-shock alone did not cause significant apoptosis in zebrafish. The mRNA expression of Wnt target genes VEGF $_{165}$ and IL-8/CXCL8) was increased when Wnt signaling was activated. However, the effect of Wnt8a stimulation was partially abolished by higher levels of Bachl expression in zebrafish (Figure 3(c)).

3.4. Bach1 Occupies the TCF/LEF-Binding Site of the VEGF Promoter and Recruits HDAC1 to the VEGF Promoter and Then Inhibits VEGF Expression in HUVECs. We previously demonstrated that Bachl interacted with TCF4 and occupied the TCF4 binding site of the IL- 8 promoter and then repressed IL-8 expression in HUVECs [14]. To further validate VEGF as a Bachl downstream target, we performed VEGF reporter assays using different VEGF reporter constructs and then investigated the effect of Bach1 overexpression on the luciferase activity of these constructs. In HEK293T cells, Bach1 overexpression significantly reduced the luciferase activity of all reporter constructs that contained the TCF/LEF-binding site (i.e., -2650, -529, and -228) (Figure $4(\mathrm{a})$ ). The results from chromatin immunoprecipitation assays indicated that Bachl bound to the region from position -105 to -301 of the VEGF promoter in HUVECs (Figure 4(b)), which contains the consensus TCF/LEF-binding site (positions -144 to -139 , Figure 4(a)), further confirming that Bach1 occupies the TCF/LEF-binding site of the VEGF promoter. We have recently shown that Bachl interacts with histone deacetylase 1 (HDACl) and recruits HDAC1 to the IL-8 Promoter in HUVECs [14]. We then asked whether the inhibitory effect of Bachl on VEGF expression is related to HDAC1. Chromatin immunoprecipitation assays indicated that HDACl's occupancy of the VEGF promoter was $\approx 3$ fold greater in AdBachl-HUVECs than in AdGFP-HUVECs, whereas the enrichment of $\mathrm{H} 3$ acetylation at these binding sites was decreased in AdBachl-HUVECs (Figure 4(c)). Furthermore, the HDAC inhibitor Trichostatin A (TSA) treatment increased the transcriptional activity of the VEGF promoter in Bachl-overexpressing HUVECs (Figure 4(d)), indicating that Bach1 may repress VEGF gene transcription by recruiting HDAC1. In addition, the results from our cellular experiments also indicated that higher levels of Bach1 expression reduced VEGF mRNA and protein levels, whereas knockdown of Bach1 expression increased VEGF mRNA and protein expression in HUVECs (Figures 5(a) and 5(b)).

\subsection{Exogenous Administration of VEGF or IL-8 Partially} Rescued Bach1-Driven Antiangiogenic Response in HUVECs. To determine the role of VEGF and IL- 8 in the regulation of 


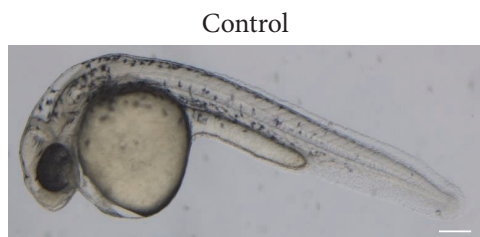

(A)

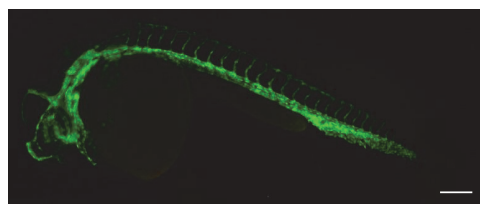

(C)

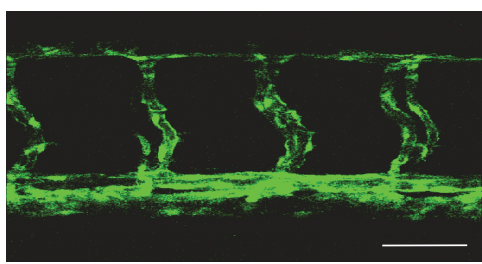

(E)

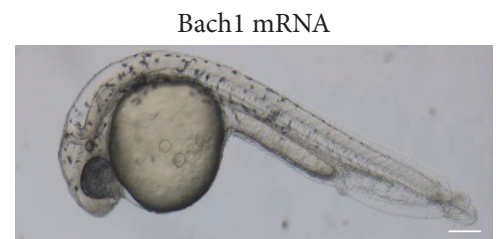

(B)

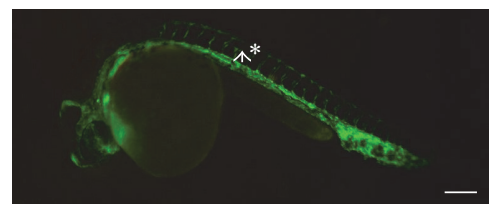

(D)

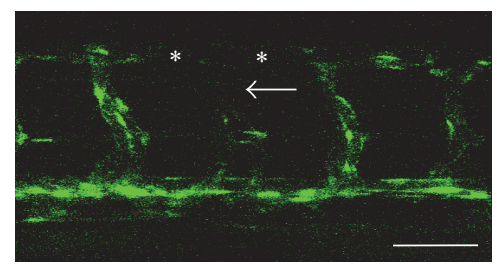

(F)

(a)

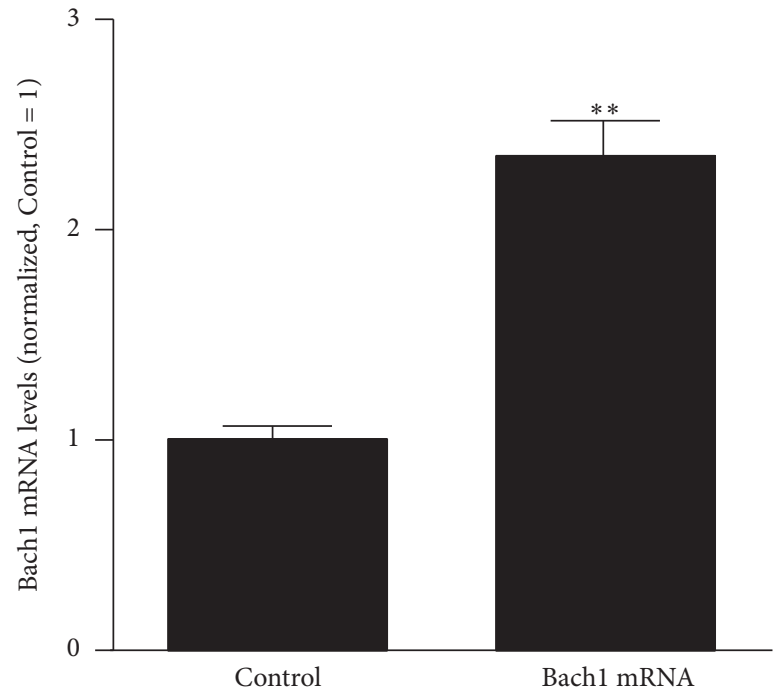

(b)

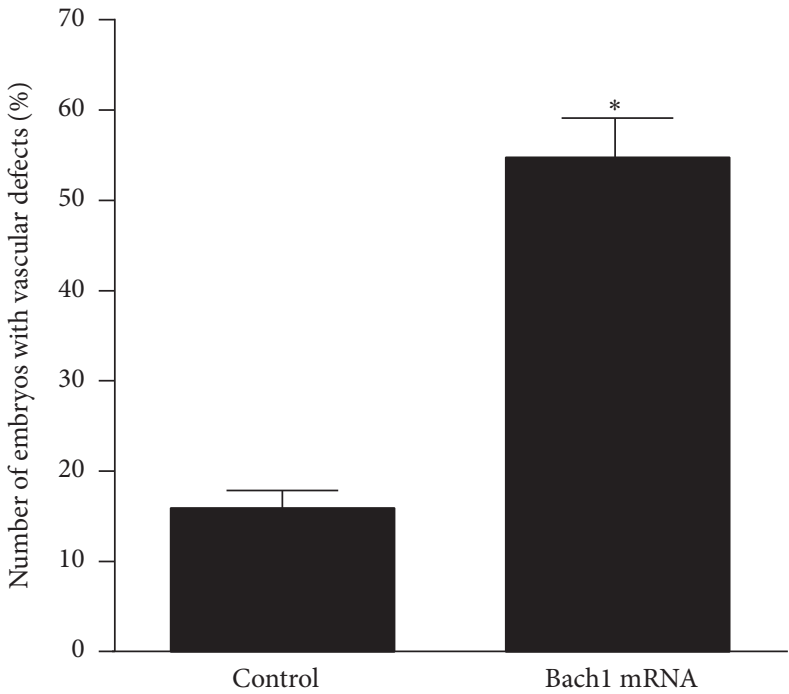

(c)

FIGURE 2: Bach1 overexpression induces vascular defects in zebrafish embryos. (a) Zebrafish vasculature was evaluated via fluorescent images (C-D) or confocal images (E and F) in normal 32-hpf Tg(fli1:EGFP) zebrafish embryos (A, C and E) and in embryos that had been injected with $200 \mathrm{pg}$ of Bach1 mRNA (B, D and F) at the single-cell stage. Bachl mRNA injections disrupted formation of the intersegmental vessels (ISV) (arrow) and the dorsal longitudinal anastomotic vessels (DLAV) (asterisks). (A-D) Scale bar: $100 \mu \mathrm{m}$. (E and F) Scale bar: $50 \mu \mathrm{m}$. (b) Bachl mRNA levels were evaluated in normal and Bachl-mRNA-injected embryos via quantitative real-time RT-PCR $\left(n=8\right.$; ${ }^{* *} P<0.01$ versus Control). (c) Embryos with defects in ISV and DLAV were counted and expressed as a percentage of the total number of embryos in each experimental group $\left(n=50 ;{ }^{*} P<0.05\right.$ versus Control).

angiogenesis by Bachl, we performed Matrigel tube-forming assays in Bachl expressing HUVECs with external VEGF or IL-8 administration. Exogenous administration of VEGF or IL-8 partially rescued Bach1-driven antiangiogenic response in HUVECs (Figure 6).

\section{Discussion}

We have shown that Bachl is implicated in ischemia-induced angiogenesis in mice [14]. However, the function of Bachl in developmental angiogenesis is not well understood. Here, we 


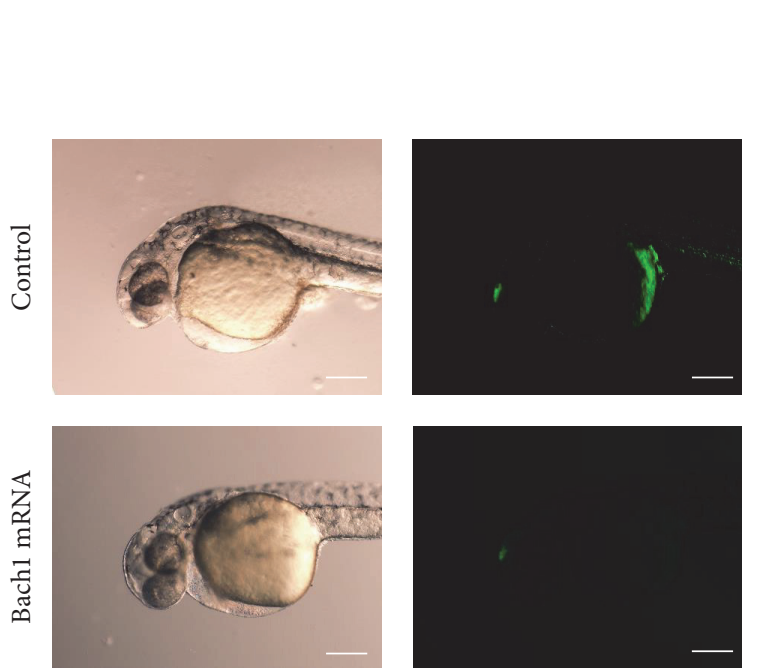

(a)

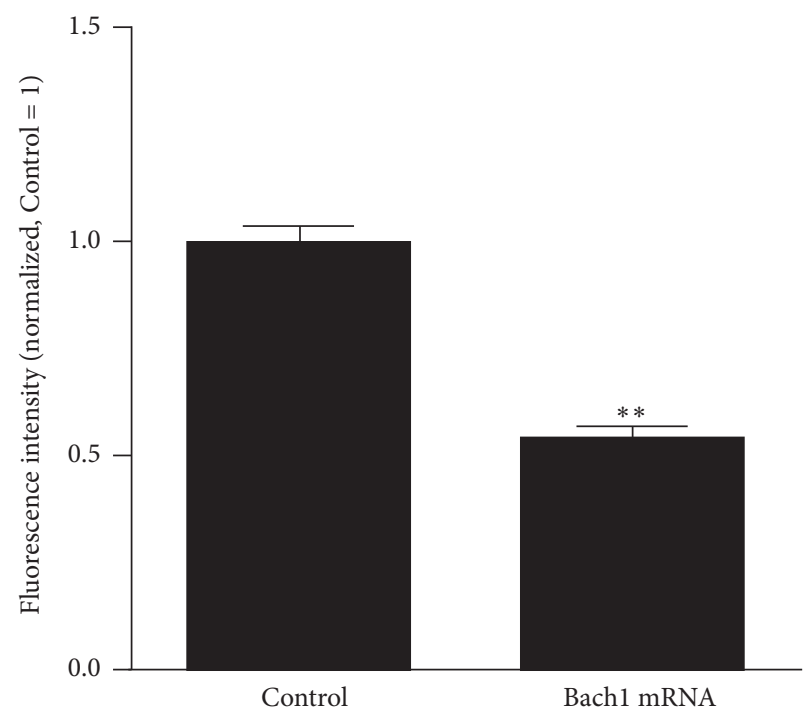

(b)

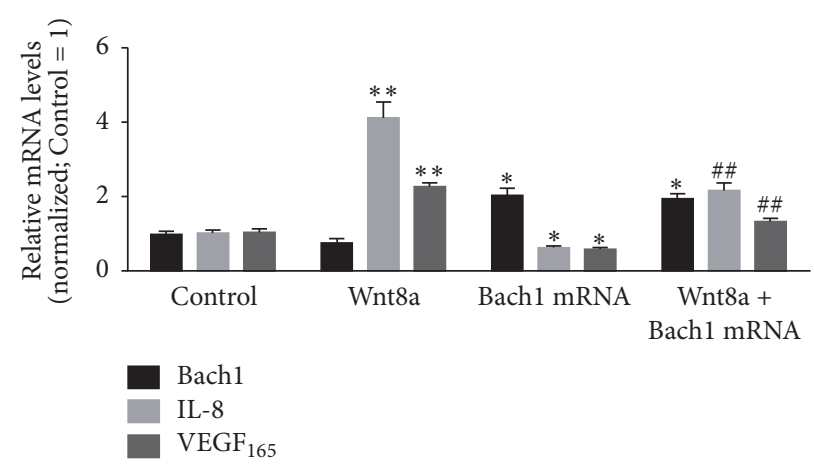

(c)

FIGURE 3: Bachl suppresses endogenous Wnt/ $\beta$-catenin signaling and exogenous Wnt8a stimulated VEGF and IL-8 gene expression in zebrafish. (a) Representative fluorescent images of 36-hpf $\operatorname{Tg}($ TOP:GFP) transgenic zebrafish embryos that had been injected with or without Bachl mRNA. (b) The fluorescence intensity of GFP was quantified and expressed as the as the ratio of measurements Bachl mRNA and Control group ( $n=12$; ${ }^{* *} P<0.01$ versus Control). (c) IL-8, VEGF ${ }_{165}$, and Bach1 mRNA levels were evaluated in 32-hpf Tg(hsp:wnt8a) embryos following Bach1 mRNA injections at the one cell stage and Wnt pathway activation (heat-shock) at $24 \mathrm{hpf}\left(n=10\right.$; ${ }^{*} P<0.05$, ${ }^{* *} P<0.01$ versus Control; ${ }^{\# \#} P<0.01$ versus Wnt8a).

found that Bach1 suppressed the developmental angiogenesis of zebrafish and that this function was associated with declines in Wnt/ $\beta$-catenin signaling and VEGF and IL-8 expression in zebrafish. Thus, Bachl functions not only in angiogenesis after ischemic injury but also in development angiogenesis.

In the zebrafish genome, there are two Bach1 homolog genes, bachla and Bachlb, which are located on chromosomes 15 and 10, respectively [17]. Bachlb contains both BTB and BZIP domains and mediates the regulatory role of heme in transcription of the zymogens in zebrafish $[17,18]$. Recent study has identified that Bachlb is expressed ubiquitously from one cell to $48 \mathrm{hpf}$ [18], and this observation is consistent with our observation. In zebrafish, the sprouting and extension of endothelial cells to form intersegmental vessels (ISV) from the dorsal aorta is considered as angiogenesis [26]. In Bachlb overexpression zebrafish, we found that the extension of ISV was dramatically hindered, suggesting that Bachlb plays a key role in the proliferation and migration of ECs during sprouting angiogenesis. In fact, our previous study has shown that Bach1 suppresses endothelial cell migration, proliferation, and tube formation in HUVECs [14]. VEGF-A is expressed in the ventromedial region of each somite during sprouting angiogenesis $[10,27]$. Similar to human, VEGF121 and VEGF165 are the two most abundant isoforms of VEGFA in the zebrafish. Zebrafish express two VEGFR2 homologs known as Kdrl/Flk1 and Kdr/Kdrb. VEGF-A/VEGFR2 signaling is required for early intersegmental vasculature development with mutation in VEGF-aa or loss of VEGFR2 homologs causing nearly complete inhibition of the formation of the ISV [26]. VEGF-A signaling has been shown to direct endothelial cells to migrate out of existing vasculature [28], and impaired endothelial migration was associated with abnormal formation of the ISV [29]. IL-8 is another key proangiogenic factor. IL-8 and its receptors CXCR1 and CXCR2 have been described in zebrafish [30], and 


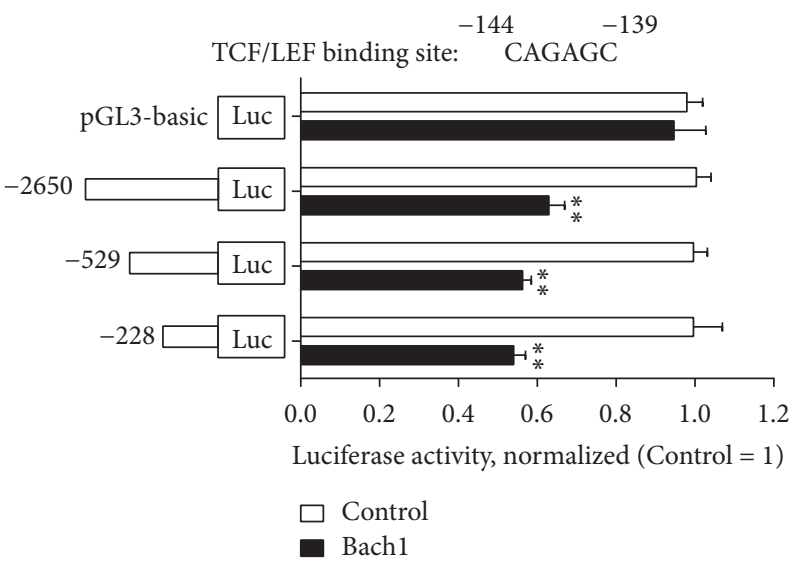

(a)

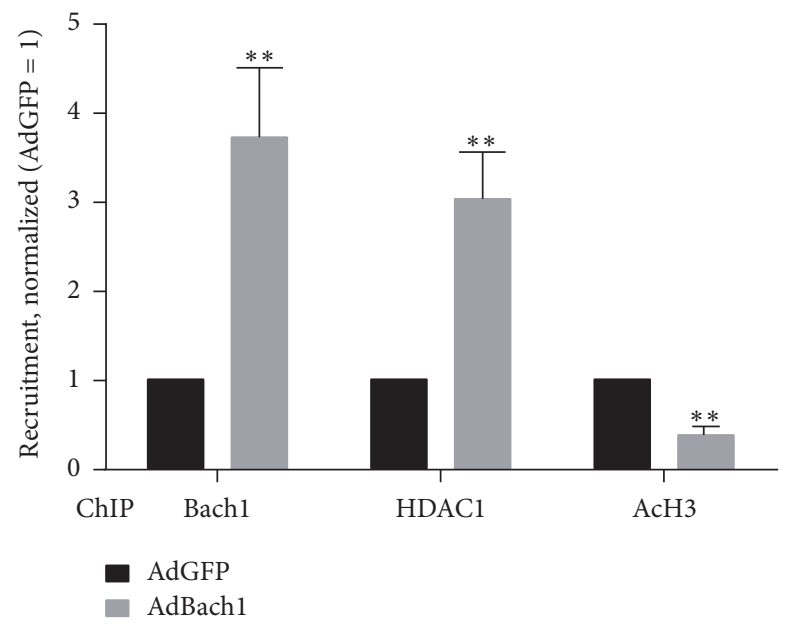

(c)

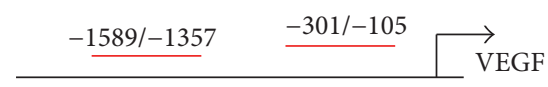

ChIP Bach1

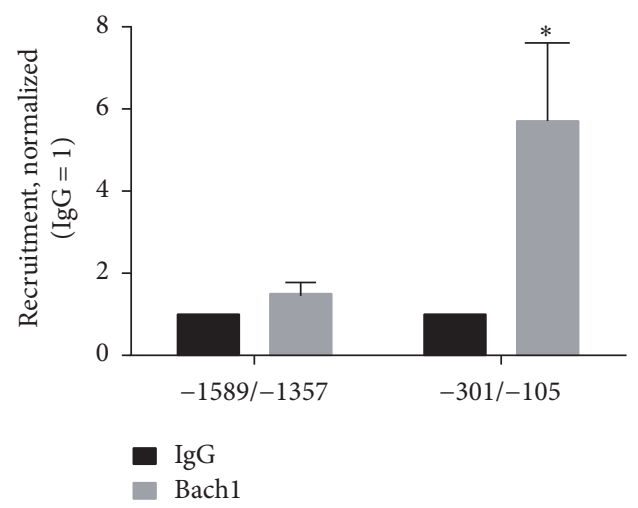

(b)

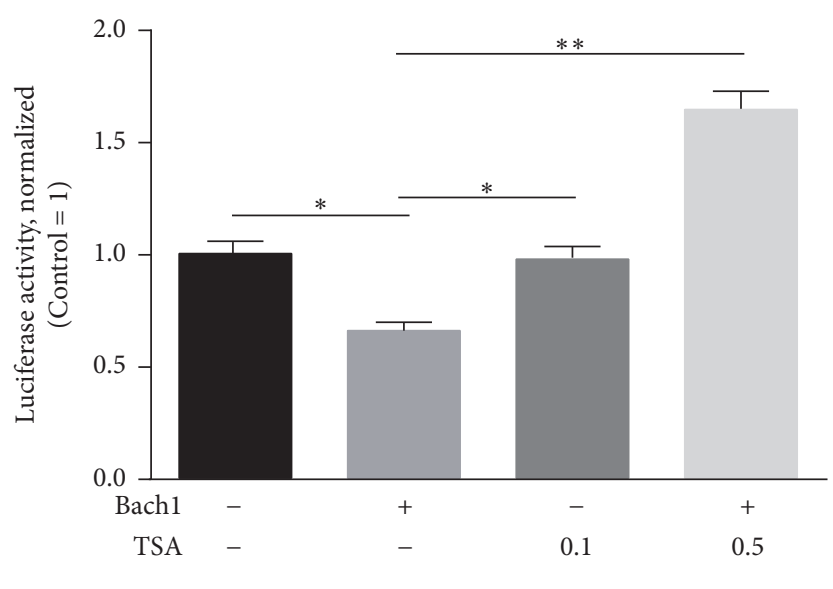

(d)

FIGURE 4: Bachl occupies the TCF/LEF-binding site of the VEGF promoter and recruits HDAC1 to the VEGF promoter in HUVECs. (a) Potential TCF/LEF-binding site of the VEGF promoter is shown. Luciferase reporter constructs were created containing the truncated (-2650, -529 , and -228) versions of the VEGF promoter; then the VEGF reporter or a pGL3-basic luciferase reporter and $\beta$-gal were cotransfected with a Bach1-coding vector or with an empty vector (Control) into HEK293T cells, and luciferase activity was evaluated 48 hours later $(n=3$; ${ }^{* *} P<0.01$ versus Control). (b) Chromatin immunoprecipitation DNA was isolated with an anti-Bachl antibody or with a Control IgG antibody, and then quantitative real-time RT-PCR analyses were performed with primer sequences from the indicated regions of the VEGF promoter in HUVECs $\left(n=4 ;{ }^{*} P<0.05\right.$ versus Control IgG). (c) HUVECs were transfected with AdGFP or AdBachl; then chromatin immunoprecipitation was performed with antibodies against the indicated proteins. Quantitative real-time RT-PCR analyses were performed with a primer for the VEGF promoter $(-301 /-105)\left(n=3 ;{ }^{* *} P<0.01\right.$ versus AdGFP). (d) HUVECs were transfected with AdGFP or AdBach1 and with the VEGF (-228) promoter construct; then, the cells were treated with or without TSA $(0.1$ or $0.5 \mu \mathrm{mol} / \mathrm{L})$ for 24 hours and luciferase activity was quantified $\left(n=3 ;{ }^{*} P<0.05,{ }^{* *} P<0.01\right)$.

silencing of IL-8 expression in zebrafish inhibited vascular development [11]. The results presented here indicate that Bachlb overexpression in zebrafish reduced VEGF $_{165}$ and IL-8 mRNA levels and exogenous VEGF-A or IL-8 was able to partially reverse Bachl-driven antiangiogenic response in HUVECs, confirming VEGF and IL-8 as the major targets of Bachl-mediated antiangiogenic functions. Thus, Bach1 appears to impair the developmental angiogenesis in zebrafish by reducing the expression of VEGF and IL- 8 and by impairment of endothelial cell migration and proliferation.
The Wnt $/ \beta$-catenin signaling pathway plays a vital role in vascular development and the process of angiogenesis [31], and the inhibition of $\beta$-catenin has been shown to suppress angiogenesis in zebrafish [32]. These observations are consistent with our results. We found that Bachl suppressed endogenous Wnt signaling in zebrafish, and this decline was associated with the decrease in the developmental angiogenesis of zebrafish embryos. Wnt target genes including VEGF and IL- 8 are transcriptionally activated by $\beta$-catenin/TCF signaling in HUVECs $[7,31]$. The gene promoter of human 


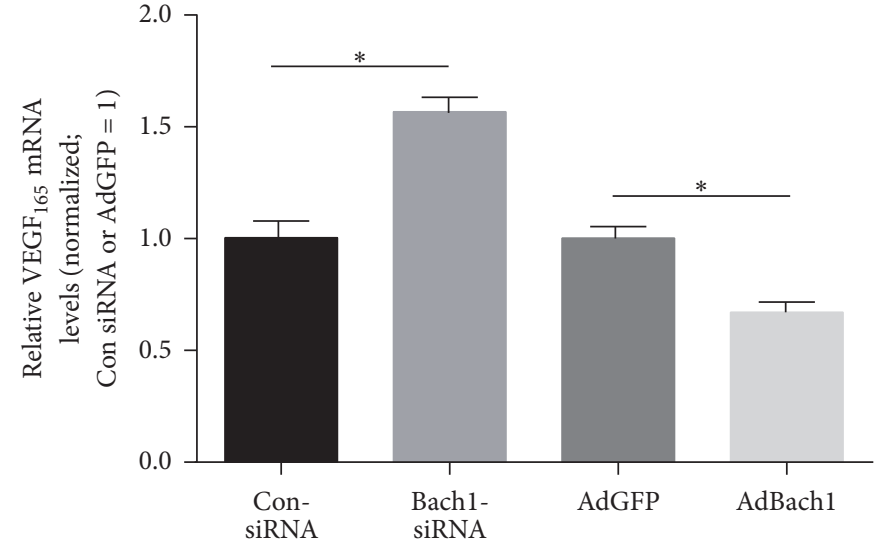

(a)

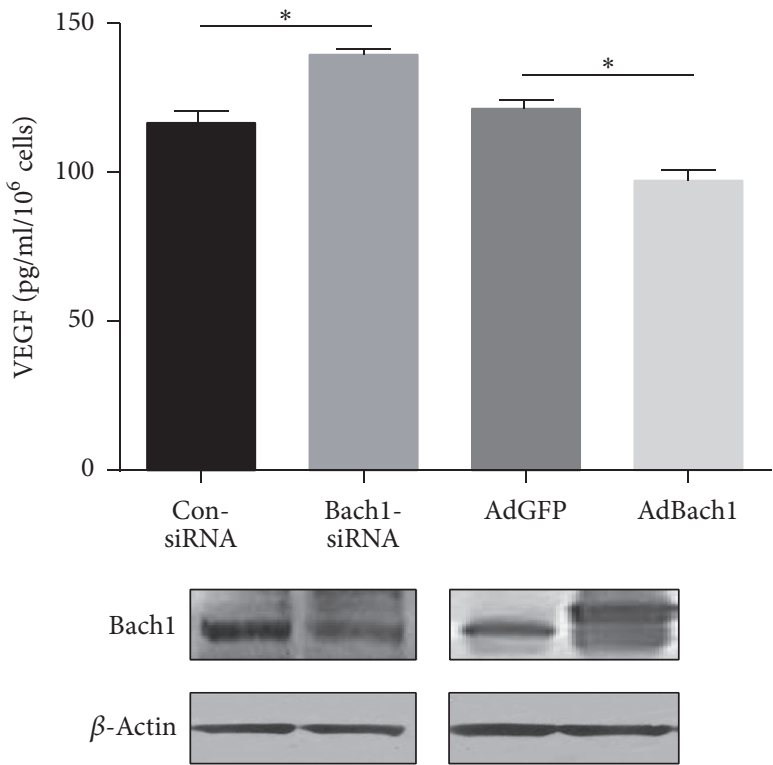

(b)

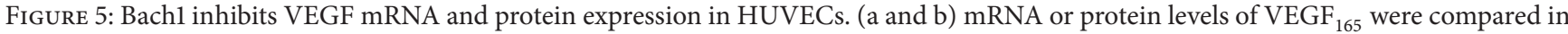
ConsiRNA- and Bach1siRNA-transfected HUVECs and in AdGFP and AdBachl-HUVECs. mRNA levels (a) were evaluated via quantitative PCR $\left(n=4 ;{ }^{*} P<0.05\right)$ and protein levels $(b)$ were evaluated via ELISA $\left(n=3 ;{ }^{*} P<0.05\right)$.
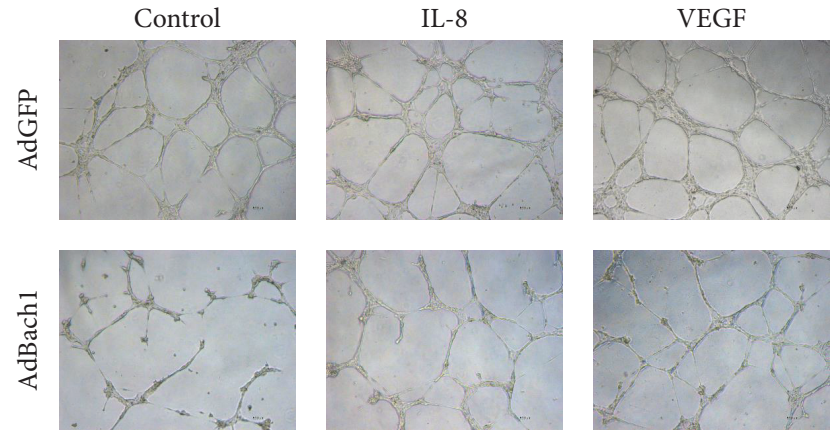

(a)

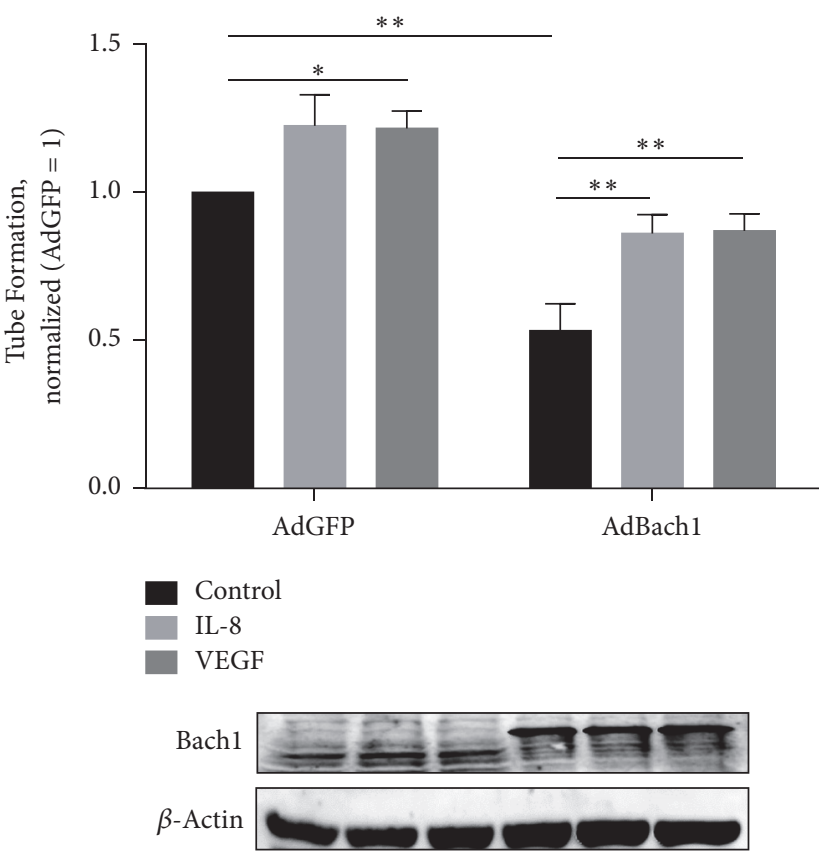

(b)

FIGURE 6: Exogenous administration of VEGF or IL-8 partially rescued Bach1-driven antiangiogenic response in HUVECs. (a) HUVECs were transfected with AdGFP or AdBach1 for 24 hours and then incubated with or without VEGF (50 ng/mL) or IL-8 (50 ng/mL) for 24 hours. Endothelial tube formation assay in Matrigel was performed in presence of VEGF or IL-8. Tube length was quantified and expressed as the fold change relative to AdGFP $\left(n=4 ;{ }^{*} P<0.05,{ }^{* *} P<0.01\right)$. Scale bar: $500 \mu \mathrm{m}$. (b) Bachl and $\beta$-actin protein levels were evaluated via Western blot. 
VEGF-A contains several consensus binding sites for $\beta$ catenin/TCF [33]. We have shown that Bachl binds directly to TCF4 and reduces the interaction of $\beta$-catenin with TCF 4 and then impairs Wnt/ $\beta$-catenin signaling in HUVECs [14]. However, the mechanisms underlying the regulation of VEGF by Bachl are mostly unknown. Our results indicated that Bachl occupies the TCF/LEF-binding site of the human VEGF promoter, and Bach1 suppresses VEGF transcription by, at least in part, recruiting HDACl to the VEGF promoter. Similar results were also obtained in the transcriptional regulation of IL- 8 by Bach1 [14]. Previous investigation has shown that Bachl inhibits oxidative stress-induced cellular senescence by recruiting $\mathrm{HDACl}$ to the promoter of a subset of p53-targeted genes [34]. Thus, HDAC1 may be a key component of the mechanism(s) by which Bachl impedes gene transcription.

Bach1 has previously been shown to inhibit Nrf2-dependent induction of heme oxygenase-1 (HO-1) gene expression $[13,35]$. Our previous study also indicates that HO-1 was repressed by Bachl during arsenite-mediated angiogenesis [36]. HO-1 has been shown to promote angiogenesis in ischemic hearts by induction of VEGF [37]. Thus, Bach1 likely inhibits angiogenesis by regulating a variety of factors, including VEGF, IL-8, and HO-1. In addition, the results from our recent investigation suggest that higher levels $(\approx 100$-fold increase in mRNA expression) of Bachl enhanced ROS production and induced apoptosis in ischemic mouse hindlimbs [15]. However, we found that a slight $(\approx 2$-fold $)$ increase in Bachlb mRNA expression did not induce the apoptosis (data not shown) in zebrafish in the present study, which suggests that the effect of Bachlb overexpression on the developmental angiogenesis in zebrafish cannot be explained by apoptosis.

In conclusion, the results presented here indicate that Bach1 suppresses the developmental angiogenesis of zebrafish and that this function is associated with declines in $\mathrm{Wnt} / \beta$ catenin signaling and VEGF and IL-8 expression. Bach1 inhibits VEGF transcription by recruiting HDACl to the VEGF promoter in HUVECs. Thus, the identification of Bachl's role in developmental angiogenesis may provide new insight into angiogenesis-based therapeutic approaches.

\section{Disclosure}

Li Jiang and Meng Yin share first authorship.

\section{Conflicts of Interest}

The authors declare no conflicts of interest.

\section{Authors' Contributions}

Dan Meng conceived the general idea and framework of the project, designed the majority of the experiments, and oversaw the project to its completion. Jianyi Zhang helped design experiments. Li Jiang and Meng Yin performed most of the experiments. Jie Xu, Mengping Jia, Shaoyang Sun, and $\mathrm{Xu}$ Wang analyzed data and assisted with experiments. Dan
Meng wrote the manuscript. Li Jiang and Meng Yin made equal contribution to this work.

\section{Acknowledgments}

The authors thank Fengdi Zhao for technical support. This work was supported by General Programs of the National Natural Science Foundation of China (91639103 and 81670450 to D. Meng and 81671833 to M. Yin), the Shanghai Municipal Health Bureau Grant of China (201640074 to D. Meng), the National Key Research and Development Plan (2016YFC1102400 to M. Yin), the Collaborative Innovation Center for Translational Medicine at Shanghai Jiao Tong University School of Medicine (TM201504 to M. Yin), and the Pudong New Area Science and Technology Development Fund Innovative Capital Projects (PKJ2016-Y33 to M. Yin).

\section{References}

[1] M. Corada, D. Nyqvist, F. Orsenigo et al., “The Wnt/ $\beta$-catenin pathway modulates vascular remodeling and specification by upregulating Dll4/notch signaling," Developmental Cell, vol. 18, no. 6, pp. 938-949, 2010.

[2] E. D. Cohen, Y. Tian, and E. E. Morrisey, "Wnt signaling: an essential regulator of cardiovascular differentiation, morphogenesis and progenitor self-renewal," Development, vol. 135, no. 5, pp. 789-798, 2008.

[3] A. V. Gore, M. R. Swift, Y. R. Cha et al., "Rspol/wnt signaling promotes angiogenesis via vegfc/vegfr3," Development, vol. 138, no. 22, pp. 4875-4886, 2011.

[4] A. Cattelino, S. Liebner, R. Gallini et al., "The conditional inactivation of the $\beta$-catenin gene in endothelial cells causes a defective vascular pattern and increased vascular fragility," Journal of Cell Biology, vol. 162, no. 6, pp. 1111-1122, 2003.

[5] H.-C. Lee, Y.-Z. Lin, Y.-T. Lai et al., "Glycogen synthase kinase 3 beta in somites plays a role during the angiogenesis of zebrafish embryos," FEBS Journal, vol. 281, no. 19, pp. 4367-4383, 2014.

[6] C. Skurk, H. Maatz, E. Rocnik, A. Bialik, T. Force, and K. Walsh, "Glycogen-synthase kinase $3 \beta / \beta$-catenin axis promotes angiogenesis through activation of vascular endothelial growth factor signaling in endothelial cells," Circulation Research, vol. 96, no. 3, pp. 308-318, 2005.

[7] L. Lévy, C. Neuveut, C.-A. Renard et al., "Transcriptional activation of interleukin- 8 by $\beta$-catenin-Tcf4," Journal of Biological Chemistry, vol. 277, no. 44, pp. 42386-42393, 2002.

[8] E. Dejana, "The role of wnt signaling in physiological and pathological angiogenesis," Circulation Research, vol. 107, no. 8, pp. 943-952, 2010.

[9] A. Li, S. Dubey, M. L. Varney, B. J. Dave, and R. K. Singh, "IL8 directly enhanced endothelial cell survival, proliferation, and matrix metalloproteinases production and regulated angiogenesis," Journal of Immunology, vol. 170, no. 6, pp. 3369-3376, 2003.

[10] D. Liang, J. R. Chang, A. J. Chin et al., "The role of vascular endothelial growth factor (VEGF) in vasculogenesis, angiogenesis, and hematopoiesis in zebrafish development," Mechanisms of Development, vol. 108, no. 1-2, pp. 29-43, 2001.

[11] S. J. Stoll, S. Bartsch, H. G. Augustin, and J. Kroll, "The transcription factor HOXC9 regulates endothelial cell quiescence and vascular morphogenesis in zebrafish via inhibition of interleukin 8," Circulation Research, vol. 108, no. 11, pp. 13671377, 2011. 
[12] T. Oyake, K. Itoh, H. Motohashi et al., "Bach proteins belong to a novel family of BTB-basic leucine zipper transcription factors that interact with MafK and regulate transcription through the NF-E2 site," Molecular and Cellular Biology, vol. 16, no. 11, pp. 6083-6095, 1996.

[13] K. Ogawa, J. Sun, S. Taketani et al., "Heme mediates derepression of Maf recognition element through direct binding to transcription repressor Bach1," EMBO Journal, vol. 20, no. 11, pp. 2835-2843, 2001.

[14] L. Jiang, M. Yin, X. Wei et al., "Bach1 represses wnt/beta-catenin signaling and angiogenesis," Circulation Research, vol. 117, no. 4, pp. 364-375, 2015.

[15] X. Wang, J. Liu, L. Jiang et al., "Bachl induces endothelial cell apoptosis and cell-cycle arrest through ROS generation," Oxidative Medicine and Cellular Longevity, vol. 2016, Article ID 6234043, 13 pages, 2016.

[16] Y. Fuse, H. Nakajima, Y. Nakajima-Takagi, O. Nakajima, and M. Kobayashi, "Heme-mediated inhibition of Bach1 regulates the liver specificity and transience of the Nrf2-dependent induction of zebrafish heme oxygenase 1," Genes to Cells, vol. 20, no. 7, pp. 590-600, 2015.

[17] S. Zhang, M. Xu, J. Huang et al., "Heme acts through the Bachlb/Nrf2a-MafK pathway to regulate exocrine peptidase precursor genes in porphyric zebrafish," Disease Models and Mechanisms, vol. 7, no. 7, pp. 837-845, 2014.

[18] Q. Luo, C. Wu, S. Sun et al., “The spatial-temporal expression and functional divergence of bach homologs in zebrafish Danio rerio," Journal of Fish Biology, vol. 88, no. 4, pp. 1584-1597, 2016.

[19] N. D. Lawson and B. M. Weinstein, "In vivo imaging of embryonic vascular development using transgenic zebrafish," Developmental Biology, vol. 248, no. 2, pp. 307-318, 2002.

[20] R. I. Dorsky, L. C. Sheldahl, and R. T. Moon, "A transgenic lef1/ $\beta$-catenin-dependent reporter is expressed in spatially restricted domains throughout zebrafish development," Developmental Biology, vol. 241, no. 2, pp. 229-237, 2002.

[21] G. Weidinger, C. J. Thorpe, K. Wuennenberg-Stapleton, J. Ngai, and R. T. Moon, "The Spl-related transcription factors sp5 and sp5-like act downstream of $\mathrm{Wnt} / \beta$-catenin signaling in mesoderm and neuroectoderm patterning," Current Biology, vol. 15, no. 6, pp. 489-500, 2005.

[22] C. B. Kimmel, W. W. Ballard, S. R. Kimmel, B. Ullmann, and T. F. Schilling, "Stages of embryonic development of the zebrafish," Developmental Dynamics, vol. 203, no. 3, pp. 253-310, 1995.

[23] D. Meng, D.-D. Lv, and J. Fang, "Insulin-like growth factor-I induces reactive oxygen species production and cell migration through Nox4 and Racl in vascular smooth muscle cells," Cardiovascular Research, vol. 80, no. 2, pp. 299-308, 2008.

[24] D. Meng, A. Mei, J. Liu et al., "NADPH oxidase 4 mediates insulin-stimulated HIF- $1 \alpha$ and VEGF expression, and angiogenesis in vitro," PLoS ONE, vol. 7, no. 10, Article ID e48393, 2012.

[25] D. Meng, X. Shi, B.-H. Jiang, and J. Fang, "Insulin-like growth factor-I (IGF-I) induces epidermal growth factor receptor transactivation and cell proliferation through reactive oxygen species," Free Radical Biology and Medicine, vol. 42, no. 11, pp. 1651-1660, 2007.

[26] S. Isogai, M. Horiguchi, and B. M. Weinstein, "The vascular anatomy of the developing zebrafish: an atlas of embryonic and early larval development," Developmental Biology, vol. 230, no. 2, pp. 278-301, 2001.
[27] A. Nasevicius, J. Larson, and S. G. Ekker, "Distinct requirements for zebrafish angiogenesis revealed by a VEGF-A morphant," Yeast, vol. 17, no. 4, pp. 294-301, 2000.

[28] A. L. Koenig, K. Baltrunaite, N. I. Bower et al., "Vegfa signaling promotes zebrafish intestinal vasculature development through endothelial cell migration from the posterior cardinal vein," Developmental Biology, vol. 411, no. 1, pp. 115-127, 2016.

[29] Y. Zhou, R. Ge, R. Wang et al., "Uxt potentiates angiogenesis by attenuating notch signaling," Development (Cambridge), vol. 142, no. 4, pp. 774-786, 2015.

[30] S. H. B. Oehlers, M. V. Flores, C. J. Hall et al., "Expression of zebrafish cxcl8 (interleukin-8) and its receptors during development and in response to immune stimulation," Developmental and Comparative Immunology, vol. 34, no. 3, pp. 352-359, 2010.

[31] M. Zerlin, M. A. Julius, and J. Kitajewski, "Wnt/Frizzled signaling in angiogenesis," Angiogenesis, vol. 11, no. 1, pp. 63-69, 2008.

[32] S. Shivanna, I. Harrold, M. Shashar et al., "The C-Cbl ubiquitin ligase regulates nuclear $\beta$-catenin and angiogenesis by its tyrosine phosphorylation mediated through the Wnt signaling pathway," Journal of Biological Chemistry, vol. 290, no. 20, pp. 12537-12546, 2015.

[33] V. Easwaran, S. H. Lee, L. Inge et al., " $\beta$-catenin regulates vascular endothelial growth factor expression in colon cancer," Cancer Research, vol. 63, no. 12, pp. 3145-3153, 2003.

[34] Y. Dohi, T. Ikura, Y. Hoshikawa et al., "Bach1 inhibits oxidative stress-induced cellular senescence by impeding $\mathrm{p} 53$ function on chromatin," Nature Structural and Molecular Biology, vol. 15, no. 12, pp. 1246-1254, 2008.

[35] Y. Zhou, H. Wu, M. Zhao, C. Chang, and Q. Lu, “The bach family of transcription factors: a comprehensive review," Clinical Reviews in Allergy and Immunology, vol. 50, no. 3, pp. 345-356, 2016.

[36] D. Meng, X. Wang, Q. Chang et al., "Arsenic promotes angiogenesis in vitro via a heme oxygenase-1-dependent mechanism," Toxicology and Applied Pharmacology, vol. 244, no. 3, pp. 291299, 2010.

[37] H.-H. Lin, Y.-H. Chen, P.-F. Chang, Y.-T. Lee, S.-F. Yet, and L.Y. Chau, "Heme oxygenase-1 promotes neovascularization in ischemic heart by coinduction of VEGF and SDF-1," Journal of Molecular and Cellular Cardiology, vol. 45, no. 1, pp. 44-55, 2008. 


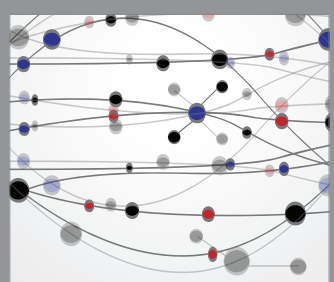

The Scientific World Journal
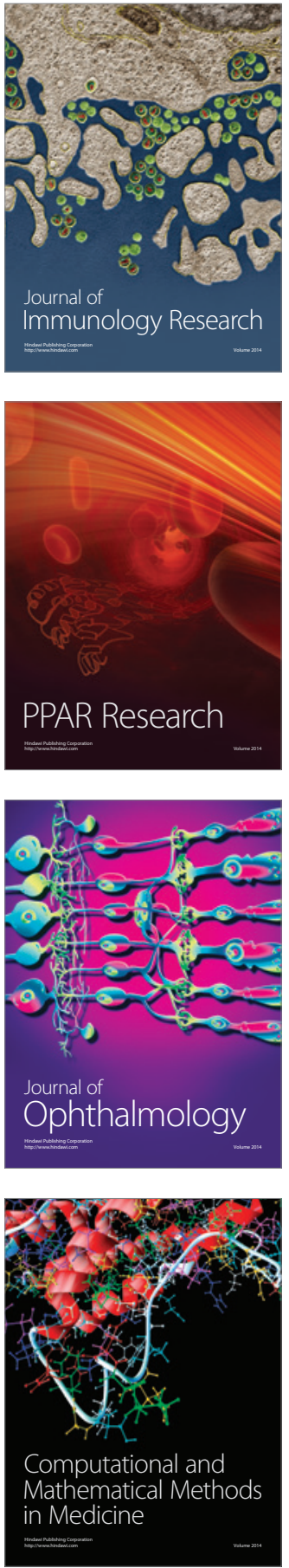

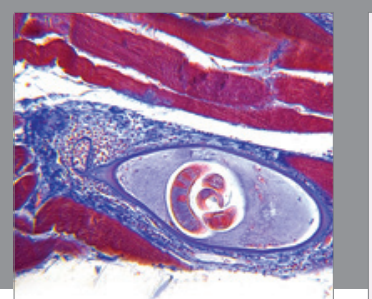

Gastroenterology Research and Practice
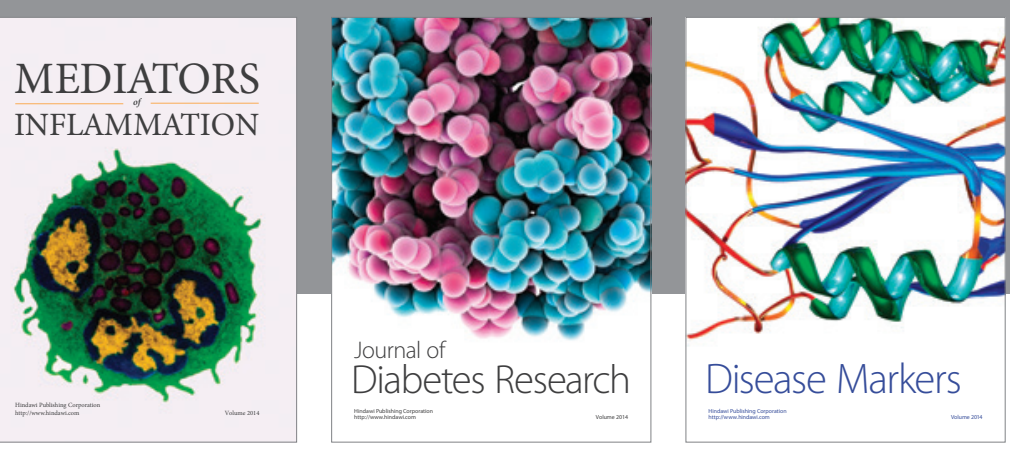

Disease Markers

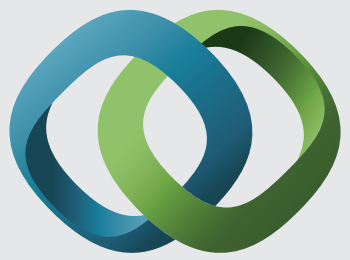

\section{Hindawi}

Submit your manuscripts at

https://www.hindawi.com
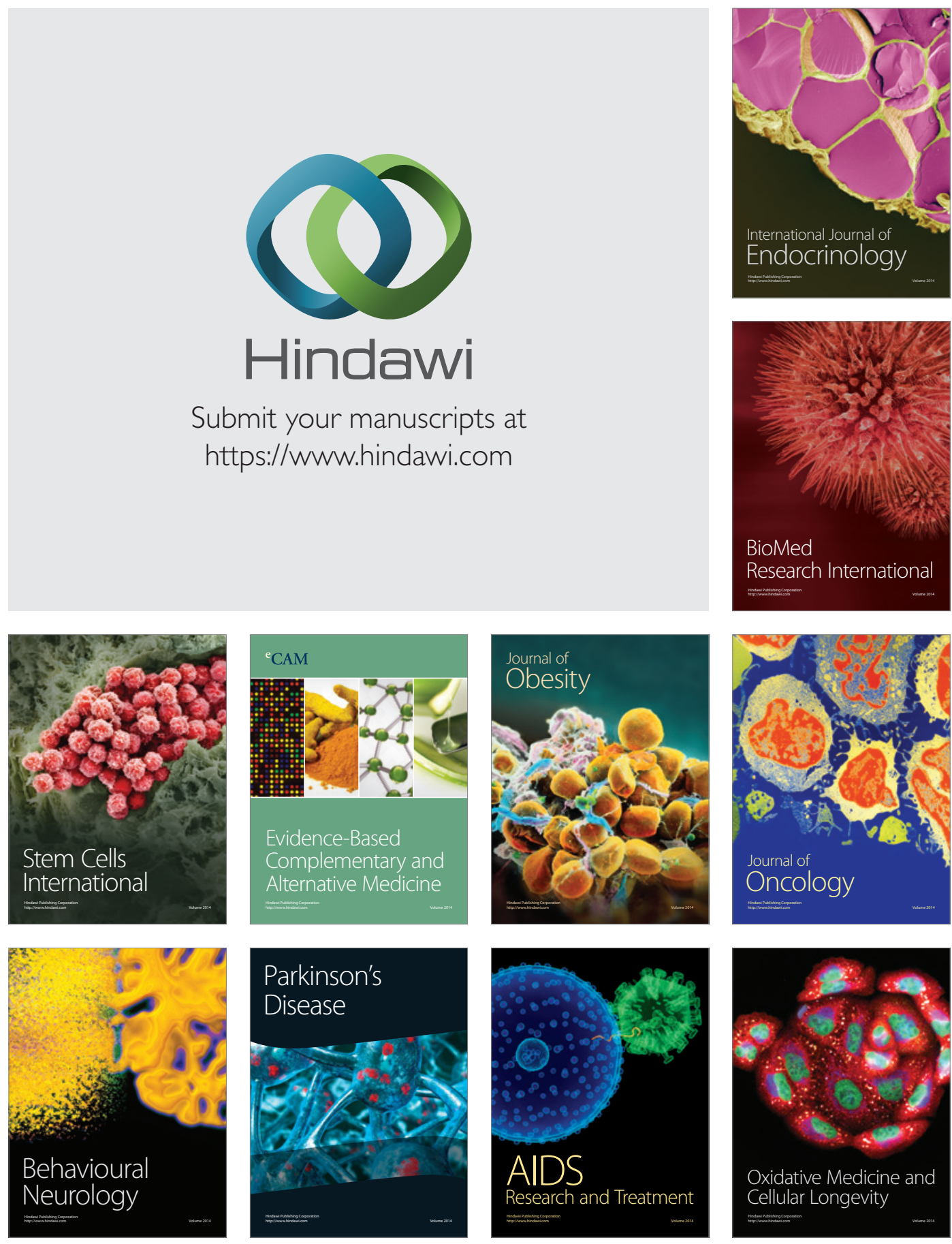